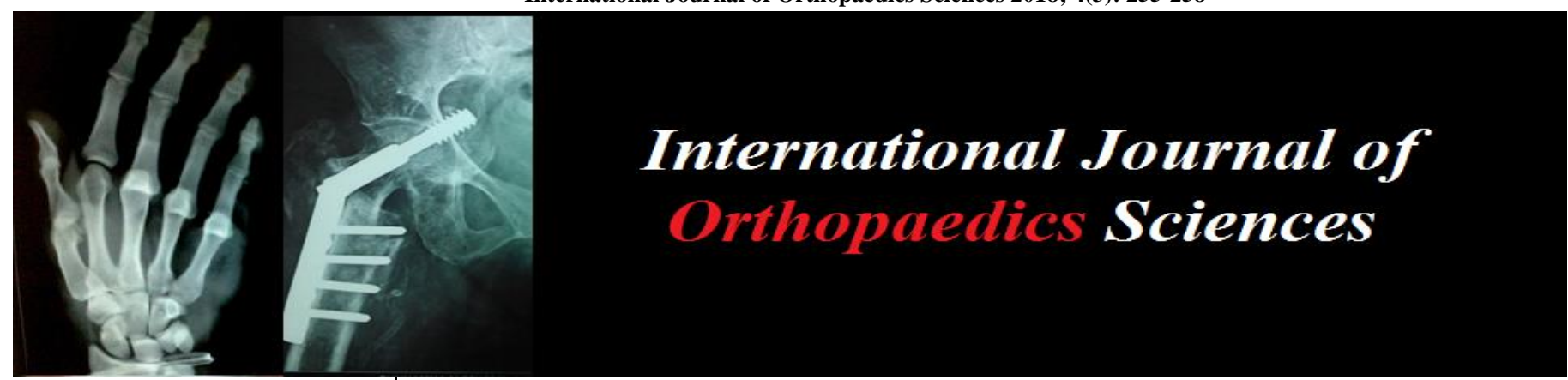

ISSN: $2395-1958$

IJOS 2018; 4(3): 255-258

(C) 2018 IJOS

www.orthopaper.com

Received: 14-05-2018

Accepted: 15-06-2018

\section{Shrestha B}

Associate Professor, HOD, Orthopedic Department,

Gandaki Medical College,

Pokhara, Nepal

\section{Sapkota K}

Assistant Professor, Manipal Hospital, HOD, Orthopedic Department, Gandaki Medical College, Pokhara, Nepal
Correspondence

Shrestha B

Associate Professor, HOD,

Orthopedic Department,

Gandaki Medical College,

Pokhara, Nepal

\section{Effectiveness of ultrasound therapy versus steroid injection in plantar fasciitis patients in western region of Nepal}

\author{
Shrestha B and Sapkota K
}

DOI: https://doi.org/10.22271/ortho.2018.v4.i3e.44

Abstract

Background: Plantar fasciitis is a common painful disorder affecting the heel and plantar aspect of the foot. There are various methods of treatment like steroid injection, nonsteroid anti-inflammatory medication, iontophoresis, laser, ultrasound, ESWT, orthotic support therapy and exercise therapies. The aim of this study is to compare the effects of ultrasound therapy versus steroid injection in plantar fasciitis patients.

Methods: This is a prospective study done on 60 patients. 30 patients were treated with single dose of local injection steroid (Group A) and 30 patients were treated with Ultrasound Therapy (Group B), 7 minutes in one session / day for 6 days /week for 2 weeks. All patients were taught plantar fascia active and passive stretching exercises to do for 10 minutes morning and 10 minutes evening at home. VAS Scores and FFI (Foot function Index) were measured.

Results: Pain Intensity was decreased in both the groups in 2 weeks and 4 weeks follow up.

VAS scores were rapidly decreased to 4.17 in Group A and 4.52 in Group B in 2 weeks follow up, which is statistically significant ( $\mathrm{p}$ value). There was statistically significant ( $\mathrm{p}$ value $<0.05$ ) in FFI (Foot function Index) in both the groups at 2 weeks and 4 weeks follow up. In Group A, FFI decreased to 30.03 \pm 1.44 in 4 weeks interval whereas it was decreased to $36.40 \pm 2.83$ in Group B in same interval.

Conclusion: Both Ultrasound therapy with stretching exercise and steroid injection with stretching exercise were effective methods for moderating pain and improving function of the foot in plantar fasciitis. It was seen that steroid injection was more superior to the Ultrasound therapy combined with exercise.

Keywords: Plantar fasciitis, VAS Scores, FFI (Foot function Index)

\section{Introduction}

Plantar fasciitis is a common painful disorder affecting the heel and plantar aspect of the foot. It is a disorder of the insertion site of tendon on the bone and is characterized by scarring, inflammation, or structural breakdown of the foot's plantar fascia. It is mostly caused due to overuse of the plantar fascia, increases in exercise, weight or age. Although plantar fasciitis was originally thought to be an inflammatory process, newer studies have demonstrated structural changes more consistent with a degenerative process ${ }^{[1,2]}$. As a result of this new observation, many in the clinical community have stated the condition should be renamed plantar fasciitis. Plantar fasciitis is the most common injury of the plantar fascia and is the most common cause of heel pain ${ }^{[3]}$.

Plantar fasciitis associated with calcaneal spur is considered to be the most common cause of foot pain in adults ${ }^{[4]}$. The etiology of plantar fasciitis is not known. It affects $10-15 \%$ of the population ${ }^{[5]}$. In $70-85 \%$ of the cases, enthesopathy of plantar fascia occurs on one side and its symptoms are always more intense on the same side. It is noteworthy that approximately $50 \%$ of individuals have had calcaneal spur and associated plantar fasciitis diagnosed ${ }^{[4]}$. Still, the correlation between plantar fasciitis and calcaneal spur is not fully understood ${ }^{[6]}$.

Risk factors that may predispose individuals to foot conditions such as calcaneal spur include age, sex, working in a standing position, Body Mass Index (BMI), uncomfortable shoes, flat foot, foot overload and a reduced range of dorsiflexion in the talocalcaneous joint. Incidence peaks in females aged 40-60 years, after menopause ${ }^{[7]}$. 
Patients usually describe pain they experience in the plantar area as burning, stabbing, dull, throbbing or sharp ${ }^{[8,9]}$. At the onset of the condition, discomfort occurs only after overloading the foot. At a later stage, rest pain or pressure pain can be experienced. Other symptoms include redness and swelling in the heel region ${ }^{[10]}$. Pain is linked to mechanical overload of the foot. Sometimes it is defined as "first-step pain" [11] since patients experience the most severe pain on taking the first step in the morning. It is a result of the tissuehealing process that occurs while sleeping. The first steps cause the rupture of tissues that fused at night. The pain tends to increase after periods of rest, after overloading the foot resulting from walking on a hard surface or carrying heavy loads, and at the end of the day ${ }^{[12]}$. The pain may also grow stronger when walking up the stairs or standing on tiptoe ${ }^{[12]}$.

Physical therapeutic procedures are most commonly applied in the conservative treatment of planter fasciitis. These include low-level laser therapy, iontophoresis, phonophoresis and shock wave therapy ${ }^{[13]}$. Ultrasound therapy is commonly used ${ }^{[13]}$. Re-educating patients and reducing their body mass are essential elements of the therapy. Foot muscle strengthening exercises, stretching exercises and soft tissue therapy are indicated as effective methods of conservative treatment ${ }^{[14]}$.

Treatment options for patients with plantar fasciitis are varied and there is little consensus on clinical approach, especially when rest is not an option. Steroid injections are a common treatment for plantar fasciitis stemming from positive evidence that they are effective at short-term management of symptoms ${ }^{[8]}$. By their nature, corticosteroids are not regenerative, are directed primarily at producing relief of pain, and have limited evidence supporting long-term treatment effectiveness ${ }^{[9]}$. Few other therapies have been as extensively researched.

Surprisingly, exercise has only received sparse attention in the medical literature, despite the fact, in one form or another, it is arguably the most common treatment approach used to address chronic heel pain. Furthermore, exercise-based treatment options for plantar fasciitis offer promise in that their focus is on restoring tissue function rather than simply symptom reduction. Histological reports from surgical biopsies of the plantar fascia do not demonstrate inflammatory infiltrates, but rather mycoid or fibrocartilaginous tissue that is representative of an aberrant remodeling process ${ }^{[15]}$. Accordingly, treatment efforts aimed at restoring ligament function and improving lower extremity coordination (to better distribute stress away from the plantar fascia) should be a priority. Both steroid injections and ultrasound therapy are common therapies, yet no study has directly compared these 2 interventions in the same study.

The aim of the study was to compare and assess the effects of steroid injection and ultrasound on pain level and functional state in patients with planter fasciitis.

\section{Methods}

Patients with a clinical diagnosis of plantar fasciitis were included in this study. The study was conducted in Fishtail Hospital, Pokhara and Gandaki Medical College, Pokhara (Western region of Nepal) during 2074.9.1 to 2075.3.30 (16 Dec 2017 to 14 July 2018). Patients who had chronic foot pain of more than 2 months durations, were not treated by any physical therapy method previously, received no local steroid injection and did not undergo any surgical procedure, not doing regular exercise and not using any special kind of shoes, were included in the study. The study was started with
70 patients. Out of them 10 patients left to do proper follow up. Hence, a total of 60 consecutive patients, 37 female $(61.7 \%)$ and 23 male $(38.3 \%)$, with the age of $51.23 \pm 10.60$ years (32-68 years), were included in this study. A written informed consent was obtained from each patient. Approval from the institutional review board was granted.

All patients were divided into 2 groups- Group A and Group B. There were 30 patients ( 21 females and 9 males) in Group A and 30 patients (16 females and 14 males) in Group B. They were assigned, based on convenient sampling for treatment.

Group A- treated with single dose of local injection steroid.

Group B - treated with Ultrasound Therapy.

\section{Local injection steroid (Group A)}

All patients of Group A were injected in minor OT room, near OPD room. Patients were positioned prone lying with knee slightly flexed and pillow was kept under the leg to be treated. $2 \mathrm{ml}$ Methyl Prednisolone Acetate (Injection Depo Medrol $40 \mathrm{mg} / \mathrm{ml}$ ) was aspirated in $3 \mathrm{cc}$ syringe and mixed with $0.5 \mathrm{ml}$ of $1 \%$ Lignocain. Under all aseptic condition, the prepared injection was inserted at affected planter fasciitis slowly.

\section{Application of Ultra Sound Therapy (Group B)}

All patients of Group B were sent to Physical Therapy Department. Patients were positioned prone lying with knee slightly flexed and pillow was kept under the leg to be treated. Ultrasound was given with continuous mode in concentric circles method using $1 \mathrm{MHZ}$ head 7 minutes in one session / day for 6 days /week for 2 weeks.

All patients were taught plantar fascia active and passive stretching exercises to do for 10 minutes morning and 10 minutes evening at home. They were asked to follow up on 2 weeks and 4 weeks interval. VAS Scores and FFI were recorded during $1^{\text {st }}$ day, 2 weeks follow up and 4 weeks follow up period.

\section{Outcome Measure}

\section{Visual Analogue Scale (VAS)}

For measuring the intensity of pain, Visual Analogue Scale was used in the patients with low back pain by determining their current pain by making a mark on a $10 \mathrm{~cm}$ visual analogue scale. The left end of the scale was labeled as "No Pain" and right end of the scale was labeled as "Worst Pain" possible. The mark was then measured to the nearest millimeters from 'No Pain' ends. The same method of VAS scores was used to measure the pain intensity in our patients.

\section{Foot Function Index}

Foot function index was designed to measure the impact of foot pathology on function in terms of pain, disability and movement restriction. The questionnaires were designed to give the information about the foot pain that had affected your ability to manage in everyday life. There were 17 questions in the chart. Patients had to score each question on a scale from 0 (no pain) to 10 (worst pain imaginable) that best described the patients foot pain over the past 1 week.

All data were analyzed by SPSS 17.0 and descriptive statistics were applied. Significant of the means were compared by using $\mathrm{T}$ test. The results were considered to be significant with p-value of $<0.05$. 


\section{Results}

Pain Intensity was decreased in both the groups in 2 weeks and 4 weeks follow up. Patients had a high VAS Scores at the time of diagnosis; Group A $(8.6 \pm 0.29)$ and Group B (8.5 \pm $0.29)$.

VAS scores were rapidly decreased to 4.17 in Group A and 4.52 in Group B in 2 weeks follow up, which is statistically significant ( $\mathrm{p}$ value $<0.05$ ). There were 5.58 points decreased in Group A and 5.01 points decreased in Group B in 2 weeks interval: $\mathrm{p}$ value $<0.05$ (Shown in Table 1).

Table 1: VAS Scores in each follow up

\begin{tabular}{|c|c|c|c|}
\hline & Group A & Group B & P value \\
\hline VAS 1 \pm SD & $8.6 \pm 0.29$ & $8.5 \pm 0.29$ & 0.21 \\
\hline VAS 2 \pm SD & $4.17 \pm 0.33$ & $4.52 \pm 0.35$ & 0.001 \\
\hline VAS 3 \pm SD & $3.02 \pm 0.12$ & $3.49 \pm 0.27$ & 0.001 \\
\hline
\end{tabular}

Note:

1-First day

2- 2 weeks follow up

3- 4 weeks follow up

There was statistically significant ( $\mathrm{p}$ value $<0.05)$ in FFI (Foot function Index) in both the groups at 2 weeks and 4 weeks follow up. In Group A, FFI decreased to $30.03 \pm 1.44$ in 4 weeks interval whereas it was decreased to $36.40 \pm 2.83$ in Group B in same interval, as shown in Table 2.

Table 2: Foot function Index in each follow up

\begin{tabular}{|c|c|c|c|}
\hline & Group A & Group B & P value \\
\hline FFI 1 \pm SD & $70.33 \pm 3.34$ & $76.16 \pm 2.05$ & 0.81 \\
\hline FFI 2 \pm SD & $40.53 \pm 2.02$ & $46.26 \pm 2.57$ & 0.001 \\
\hline FFI 3 \pm SD & $30.03 \pm 1.44$ & $36.40 \pm 2.83$ & 0.001 \\
\hline
\end{tabular}

There were no complications, like infections, hyper sensitivity reaction or others, encountered in any patients.

\section{Discussion}

The main medical problem in plantar fasciitis is pain. Pain is the basic symptom and the main reason why patients visit doctors. Therefore, researchers who put forward various treatment methods usually evaluate the effectiveness of a given therapy in terms of pain relief. Pain is a subjective phenomenon that is difficult to measure. Researchers categories pain assessment criteria as subjective (scales, questionnaires) and objective (evaluated indirectly on the basis of gait function analysis, muscle torque measurements, range of motions and functional tests in different positions that increase foot load). Subjective assessment of pain intensity is linked to the problem of using various pain scales, which makes it much more difficult to compare research findings and draw proper conclusions. Visual Analogue Scale (VAS) is the most popular scale. It was used, e.g., by Boerner E. et al., Jasiak- Tyrkalska E. et al., Straburzyńska-Lupa A. et $a l$, and Digovanni B.F et al. ${ }^{[15]}$ Moreover, Lukowicz M. et al. used the modified Laitinen scale, while Formosa et al. applied LEFS (Lower Extremity Functional Scale) ${ }^{[16]}$. Boerner E. et al. ${ }^{[15]}$ compared analgesic effects of 0.8 and 1.2 $\mathrm{W} / \mathrm{cm}^{2}$ ultrasound waves. When wave intensity of $0.8 \mathrm{~W} / \mathrm{cm}^{2}$ was applied, pain intensity decreased by over $71 \%$, while in the case of $1.2 \mathrm{~W} / \mathrm{cm}^{2}$ wave intensity, it was reduced by $66 \%$. The same parameters were used in our study to measure the pain intensity of the patients.

The overall incidence rate of plantar fasciitis was 10.5 per 1000 population. Compared with men, women had a significantly increased incidence rate ratio for plantar fasciitis of 1.96 (95\% confidence interval, 1.94 to 1.99$)$. The adjusted incidence age is between 30 to 70 years old. Increased body weight and body mass index have been shown to be significant risk factors for plantar fasciitis with a BMI more than $30 \mathrm{~kg} / \mathrm{m}^{2}$ having an odds ratio of $5.6(95 \%$ confident interval, 1.9 to $16.6 ; \mathrm{p}<0.01)$ compared with BMI of less than $25 \mathrm{~kg} / \mathrm{m}^{2}$.

Despite the conservative methods that are used during therapies including steroid injection, nonsteroid antiinflammatory medication, iontophoresis, laser, ultrasound, ESWT, orthotic support therapy and exercise therapies [17], there is no consensus about the most successful treatment method ${ }^{[18]}$. Therefore, our study is unique since we organized it to compare injection steroid and Ultra Sound therapies, easily applicable to our society, applied in combination with the exercise therapy for Planter fasciitis treatment.

Jasiak-Tyrkalska B. et al. ${ }^{[19]}$ proved that ultraphonophoresis (ultrasound therapy with ketoprofen gel applied as a couplant) produced better effects than the application of paraffin as a couplant. Pain intensity decreased by 4.2 points in the group treated with phonophoresis and by 2.8 points in the group exposed to ultrasound waves. Our study showed that pain intensity decreased by 5.58 points in 4 weeks interval in Group A whereas by 5.01 points in Group B in same period. However, according to Crawford F. et al. ${ }^{[20]} 0.5 \mathrm{~W} / \mathrm{cm} 2$ ultrasound wave is as effective as a placebo. Łukowicz M. et al. ${ }^{[21]}$ compared the effectiveness of phonophoresis and lowlevel laser therapy. In the case of the ultrasound therapy, pain intensity decreased by 2.4 points. In our study, the ultrasound therapy combined with stretching exercises resulted in the reduction of pain intensity by 5.01 points in 4 weeks interval. Prakash S. et al. [22] compared the ultrasound therapy combined with plantar fascia and calf muscles stretching with the foot manual therapy only and proved that both approaches demonstrated similar benefits. The authors noted improvements in pain by 4.26 (manual therapy combined with physical therapy and exercises) and by 5.80 (manual therapy combined with exercises). The fact that their groups displayed greater pain reductions than patients in the present study, may be associated with an implementation of several manual therapy approaches as well as higher-intensity ultrasound therapy.

Some groups have reported on the short- and medium term effectiveness of corticosteroid injections (without ultrasound guidance) at reducing pain in a population with chronic heel pain ${ }^{[23]}$. Both, Genc et al. ${ }^{[24]}$ and Kane et al. ${ }^{[25]}$ found a decrease in fascial thickness poststeroid (methylprednisone and triamcinolone hexacetonide, respectively) injection but do not report changes to any other sonographic feature. The present study had not included the fascial thickness but substantial changed in daily activities due to reduction in pain in 2 weeks and 4 weeks interval.

Another important problem is that long-term effects have not been evaluated. The longest term results were analyzed by DiGiovanni B.F. et al. [26] who carried out a follow-up evaluation 24 months post therapy, and Grecco M.V et al. ${ }^{\text {[27] }}$ who investigated outcomes 12 months post therapy. The other authors controlled effects immediately after therapy or just a few weeks post therapy. Our findings and literature review did not provide the answer to the question of which method produced the most effective long-term effects. It may indicate that both therapies were equally effective or that sample sizes were small. If the ultrasound therapy combined with exercises are useful in terms of analgesic effects, other benefits need to be found to help choose a suitable therapy (e.g. the cost, 
treatment duration, availability of procedures, equipment maintenance costs or treatment contraindications). Due to the fact that it is a pilot study, comparative research will be continued on a larger sample size bearing in mind long-term post therapy effects on pain intensity.

\section{Conclusion}

As a result of our study, both Ultrasound therapy with stretching exercise and steroid injection with stretching exercise were effective methods for moderating pain and improving function of the foot in plantar fasciitis. It was seen that steroid injection was more superior to the Ultrasound therapy combined with exercise. Nevertheless, it was concluded that further studies needed to be conducted for comparing the applications of Ultrasound therapy and the steroid injection given with various dosages, diagnoses, and consisting of a combination of more different therapy modalities and using wider samplings.

\section{References}

1. Cornwall MW, McPoil TG. Plantar fasciitis: etiology and treatment. Journal of Orthopaedic \& Sports Physical Therapy. 1999; 29:756-60.

2. Sammarco GJ, Helfrey RB. Surgical treatment of plantar fasciitis. Foot \& Ankle International. 1996; 17:520-6.

3. Kwong PK, Kay D, Voner RT, White MW. Plantar fasciitis. Mechanics and pathomechanics of treatment. Clinics in Sports Medicine. 1988; 7:119-26.

4. Formosa G, Smith G. Transverse frictional massage for plantar fasciitis: a clinical pilot trial in Musculoskelet Med. 2011; 33(3):107-114.

5. Renan-Ordine R, Alburqerque-Sendin F, Rodrigues De Souza DP, Cleland JA, De-Las-Penas CF. Effectiveness of Myofascial Trigger Point Manual Therapy Combined With a Self-Stretching. Protocol for the Management of Plantar Heel Pain: A Randomized Controlled Trial. J Orthop Sports Physiotherapy. 2011; 41(2):43-50.

6. Toumi. Changes in prevalence of calcaneal spurs in men \& women: A random population from a trauma clinic. BMC Musculoskel Dis. 2014; 15:87-92.

7. Johal KS, Milner SA. Plantar fasciitis and the calcaneal spur: Fact or fiction? J Foot Ankle Surg. 2012; 18:39-41.

8. Ball EM, McKeeman HM, Patterson C. Steroid injection for inferior heel pain: a randomised controlled trial. Ann Rheum Dis. 2013; 72:996-1002.

9. Crawford F, Thomson C. Interventions for treating plantar heel pain. Cochrane Database Syst Rev. 2003; 3:CD000416

10. Roxas M. Plantar Fasciitis: Diagnosis and Therapeutic Considerations. Altern Med Rev. 2005; 10:83-93.

11. Straburzyńska-Lupa A, Kornacka A, Zastosowanie ultradźwięków w, leczeniu tzw. ostrogi piętowej w, świetle badań własnych. Orthop Traumatol Rehab. 2005; 7(1):79-86.

12. Koca. Painful plantar heel spur treatment with CO-60 teletherapy: factors influencing treatment outcome. Springer Plus. 2014; 3:21-24.

13. Barrett SJ, O'Malley R. Plantar fasciitis and other causes of heel pain. Am Fam Physician. 1999; 59:2200-2206.

14. Sweeting D, Parish B, Hooper L, Chester R. The effectiveness of manual stretching in the treatment of plantar heel pain: A systematic review. J Foot Ankle Res. $2011 ; 4: 19-31$.

15. Lemont H, Ammirati KM, Usen N. Plantar fasciitis: A degenerative process (fasciosis) without inflammation. J
Am Podiatr Med Assoc. 2003; 93:234-237.

16. Renan-Ordine R, Alburqerque-Sendin F, Rodrigues De Souza DP, Cleland JA, De-Las-Penas CF. Effectiveness of Myofascial Trigger Point, Manual Therapy Combined With a Self-Stretching. Protocol for the Management of Plantar Heel Pain: A Randomized Controlled Trial. J Orthop Sports Phys Ther. 2011; 41(2):43-50.

17. Greve JM, Grecco MV, Santos-Silva PR. Comparison of radial shockwaves and conventional physiotherapy for treating plantar fasciitis. Clinics (Sao Paulo). 2009; 64:97-103.

18. Rompe JD. Shock wave applications in musculoskeletal disorders. Stuttgart (Germany): Thieme, 2002.

19. Koca. Painful plantar heel spur treatment with co-60 teletherapy: factors influencing treatment outcome. Springer Plus. 2014; 3:21-24.

20. Crawford F, Snaith M. How effective is therapeutic ultrasound in the treatment of heel pain?. Ann Rheum Dis. 1996; 55:265-267.

21. Łukowicz M, Weber-Rajek M, Ciechanowska K, Włodarkiewicz A. Ocena skuteczności laseroterapii niskoenergetycznej i fonoforezyw leczeniu objawów ostrogi piętowej. Acta Bio-Opt Inf Med. 2009; 15(4):340343.

22. Prakash S, Misra A. Effect of manual therapy versus conventional therapy in patients with plantar fasciitis - a comparative study. Int J Physiother Res. 2014; 2(1):37882.

23. Kalaci A, Cakici H, Hapa O. Treatment of plantar fasciitis using four different local injection modalities: a randomized prospective clinical trial. J Am Podiatr Med Assoc. 2009; 99:108-113.

24. Genc H, Saracoglu M, Nacir B. Long-term ultrasonographic follow-up of plantar fasciitis patients treated with steroid injection. Joint Bone Spine. 2005; 72:61-65.

25. Kane D, Greaney T, Shanahan M. The role of ultrasonography in the diagnosis and management of idiopathic plantar fasciitis. Rheumatology. 2001; 40:1002-1008.

26. Rompe JD. Plantar Fascia-Specific Stretching versus Radial Shock-Wave Therapy as initial Treatment of Plantar Fasciopathy. J Bone Joint Surg Am. 2010; 92:2514-2522.

27. DiGiovanni BF. Plantar Fascia-Specific Stretching Exercise Improves Outcomes in patients with Chronic Plantar Fasciitis. J Bone Joint Surg. 2006; 10:1775-1781. 